American Journal of Environmental Sciences 7 (5): 468-476, 2011

ISSN 1553-345X

(C) 2011 Science Publications

\title{
Evaluation of Hydrogen/Oxygen Release Compounds for the Remediation of VOCs
}

\author{
Fiore, S. and M.C. Zanetti \\ DITAG, Politecnico di Torino, Corso Duca, Degli Abruzzi 24, 10129 Torino, Italy
}

\begin{abstract}
Problem statement: Volatile Organic Compounds (VOCs) were widespread in groundwater of industrialized areas and in situ remediation intervents characterized by a high environmental compatibility were of main interest. The scope of this study was the evaluation of the potential of two innovative reagents (HRC and ORC from Regenesis) for the remediation of Volatile Organic Compounds (VOCs). The reagents respectively perform reduction and oxidation mechanisms, both effective in the degradation of VOCs. Approach: Hydrogen Release Compound (HRC) and Oxygen Release Compound (ORC) were tested about the degradation of Benzene, Toluene, Ethylbenzene and Xylene (BTEX) and some Chlorinated Aliphatic compounds (CAHs). Five series of batch tests were performed with an artificial polluted aqueous phase and some soil coming from a polluted site in which natural attenuation of VOCs occurs. Results: ORC exhibited a good efficiency in degradation of BTEX and zero order model was found as a reliable approximation of experimental data (with the exceptions of benzene and toluene, for which a first order kinetic model was trustworthy), while HRC showed a good efficiency in the degradation of CAHs and a first order model consistently estimated almost all experimental data. The experimental data were modeled by means of different mathematical equations, considering zero and first order kinetics and the results were discussed and compared. Conclusions: On the grounds of the performed tests, Oxygen Release Compound (ORC) is effective in BTEX degradation and Hydrogen Release Compound (HRC) in CAHs removal.
\end{abstract}

Keywords: Groundwater remediation, volatile organic compounds, chlorinated aliphatic compounds, hydrogen release compound, oxygen release compound

\section{INTRODUCTION}

Biodegradation of organic pollutants may happen by means of direct metabolic or co-metabolic processes: In the first the organic pollutant acts as a substrate for microorganisms, in the second the organic pollutant is degraded by microorganisms which are involved in other reactions and use other compounds as substrates. The substrates concerned in these kind of co-metabolic processes are usually short chain aliphatic hydrocarbons and mono-cyclic aromatic hydrocarbons (McCarty, 1993; Hazen, 2009).

Degradation processes based on the direct metabolism of pollutants generally concern hydrocarbons with less than 20-25 Carbon atoms in their molecule, Benzene, Toluene, Ethylbenzene and Xylene (BTEX) (Farhadian et al., 2008) and Polynuclear Aromatic Hydrocarbons (PAH). Cometabolic degradative processes usually involve
Chlorinated Aliphatic Hydrocarbons (CAHs), Polychlorobiphenils (PCB) and pesticides. Aerobic cometabolism is effective in medium/low CAHs degradation (Frascari et al., 2006; 2007), although for some of these pollutants (i.e., chlorobenzene, vinyl chloride) microbic species able to play a direct metabolic degradation are available. The aerobic cometabolisms of highly-chlorinated CAHs (i.e., perchloroethylene, PCE, carbon tetrachloride, hexachlorobenzene) is usually inhibited and it happens only with the support of a strong oxidant, therefore the preferential degradation mechanism is anaerobic cometabolism (McCarty, 1993).

The anaerobic degradation of CAHs may concern several reactions: Hydrolysis, dehydroalogenation, dichloroelimination and reductive dechlorination. All the above cited mechanisms require reductive conditions, often occur in a sequence and may be supported by microorganisms or abiotic (Ferguson and Pietari, 2000). 
Am. J. Environ. Sci., 7 (5): 468-476, 2011

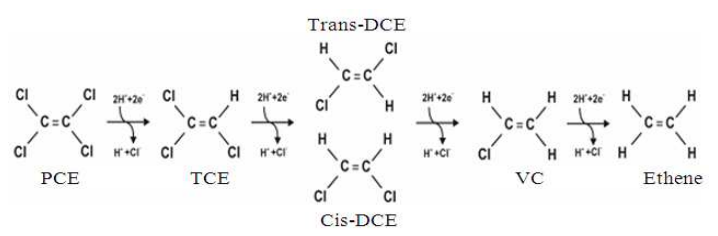

Fig. 1: Reductive dechlorination of perchloroethylene (PCE)

Reductive dechlorination, which is able to promote the growth of microbic population specific for CAHs degradation (i.e., Dehalococcoides, Sulfurospirillum multivorans, Dehalobacter restrictus) (Robinson et al., 2009) is the mechanism regularly applied to the remediation of sites polluted by organic chlorinated compounds, both considering natural attenuation and engineered solutions such as enhanced bioremediation, based on the addition of electron donors and/or nutrients to groundwater. Dehalococcoides microbic populations are able to use hydrogen as an electron donor towards a Complete reductive dechlorination of CAHs, otherwhile different populations usually produce an incomplete dehalogenation of the pollutants (Aulenta et al., 2005).

Reductive dechlorination typically concerns the biodegradation of highly substituted CAHs (i.e., PCE and Trichloroethylene, TCE) and involves the substitution of a chlorine atom with a hydrogen atom: hydrogen, that is a reducing agent, plays the role of the electron donor and pollutants act as electron acceptors. Anaerobic reductive dechlorination of $\mathrm{CAH}$ removes a chlorine atom from the pollutant molecule, produces a mole of hydrochloric acid at each step and has ethene as final product Fig. 1 (McCarty, 1993). Vinyl Chloride (VC), due to its carcinogenicity, is the secondary product of main concern. The produced hydrochloric acid reacts with bicarbonate to produce carbon dioxide, thus further decreasing groundwater $\mathrm{pH}$ (Robinson et al., 2009).

The complete dehalogenation of CAHs to ethene is generally inhibited by several issues:

- Electron donors scarceness (Yang and McCarty, 2002; Aulenta et al., 2006; 2007): It may be bypassed by the addiction of organic biodegradable substrates. Molecular hydrogen is the most important electron donor in reductive dehalogenation processes and its generation in situ remediation processes is usually due to the degradation of organic substrates, thus defined indirect electron donors, by means of fermentative processes. The influence of specific organic substrates on the composition of the supported microbial population was recently investigated (Azizian et al., 2010)

- Abundance of competing electron acceptors (mainly nitrates, sulfates, iron and manganese)
(Robinson et al., 2009; Yang and McCarty, 1998; Heimann et al., 2005): Actually the evaluation of the stimulating effect of indirect electron donors not only about dechlorinative microorganisms but also on competing populations, according to the thermodynamic sequence nitrates reductive $>$ iron and manganese reductive $>$ sulfate reductive > methanogenic $>$ dechlorinative, has a primary importance

- Occurrence of acidic conditions despite of the buffering effect of groundwater alkalinity (Robinson et al., 2009; McCarty et al., 2007) and of soil mineralogy (particularly calcite, iron oxides and gypsum minerals) (Robinson et al., 2009; Lee and Batchelor, 2004). The optimal $\mathrm{pH}$ for reductive dechlorination is between 6.8 and 7.8 (Middeldorp et al., 1999; Cope and Hughes, 2001), therefore in situ alkalinity value influences groundwater buffering ability and consequently the extent of dechlorination (McCarty et al., 2007; Adamson et al., 2004) particularly of lesser chlorinated compounds such as 1,2-dichloroethene, 1,2-(DCE) and VC (Christ et al., 2005)

- Highly substituted CAHs are characterized by a reductive dechlorination kinetic more favorable if compared to their degradation products (Ferguson and Pietari, 2000; Borum, 2002; EPA, 2009): It is common in the field the lack of proofs of a complete CAHs degradation, therefore 1,2-DCE and $\mathrm{VC}$ are often detected in polluted sites

- Absence of a microbial population specific for the dechlorination of each pollutant, particularly DCE and VC (Robinson et al., 2009; Borum, 2002; Amos et al., 2007)

- Insufficient contact time between electron donors and acceptors (Silva et al., 2006): In the field this condition happens in sites characterized by a high seepage velocity (Borum, 2002)

CAH biodegradation, particularly of lesser substituted compounds, may also involve oxidation processes (McCarty, 1993) in which the pollutant acts as the electron donor and oxygen as the electron acceptor. These processes lead to unstable degradation intermediates (i.e., epoxides), that are rapidly converted into high biodegradable not chlorinated compounds, such as alcohols and organic acids, with the release of chloride ions. The most common mechanisms, all involving the exchange of two electrons for each pollutant's molecule, are $\alpha$-hydroxylation (substitution of a $\mathrm{H}$ with a $\mathrm{OH}$ ), halo-oxidation (creation of a halogen-oxygen bond), epo-oxidation (creation of epoxides from unsaturated chlorinated hydrocarbons) (Grunanger and Finzi, 1997). 
In this study the results of some laboratory batch tests, aimed to the evaluation of the potential of two Reagents ( $\mathrm{HRC}$ and ORC from Regenesis) for the remediation of Volatile Organic Compounds (VOCs), are discussed. The experimental data were obtained from five series of batch tests performed with an artificial polluted aqueous phase and some soil coming from a polluted site in which natural attenuation of VOCs occurs. BTEX and some benzenic VOCs were considered as pollutants and HRC as remediation reagent in a first test, $\mathrm{BTEX}$ and some CAHs were considered as pollutants and $\mathrm{HRC}$ as remediation reagent in a second test, BTEX and some CAHs were considered as pollutants and ORC as remediation reagent in a third test. The net contribute of the soil in removing the pollutants from the aqueous phase by means of sorption processes was evaluated in two separate tests, in which the same pollutants (BTEX and some aromatic VOCs and BTEX and some CAHs) were considered without any remediation reagent. The experimental data gathered from the batch tests underwent regression by means of different mathematical equations, modeling a zero and a first order kinetic and the results were discussed and compared.

The hydrogen/oxygen release compounds: Two reagents that may be employed in enhanced bioremediation processes are tested in this study towards BTEX and other VOCs, particularly aromatic hydrocarbons and CAHs: Hydrogen Release Compound (HRC, a readily biodegradable organic substrate, mainly made of lactate, that acts as an indirect electron donor) and Oxygen Release Compound (ORC), containing magnesium peroxide.

Hydrogen Release Compound (HRC) is a patented mixture of polylactate and glycerol, characterized by a density equal to $1.3 \mathrm{~kg} \mathrm{dm}^{-3}$ and a viscosity equal to $2 \cdot 10^{5}$ centipoise at room temperature. It may be considered an innovative reagent for groundwater remediation, particularly for the removal of chlorinated volatile compounds by means of enhanced bioremediation, since it acts as an indirect electron donor. HRC, which appears as an amber molasse, may be employed by direct injection in groundwater or in a permeable reactive barrier.

Polylactate in HRC, added to an aqueous phase, is hydrolyzed to lactic acid which is biologically degraded by acetogenic anaerobic microorganism to short chain volatile acids (mainly pyruvic and acetic acids), with the release of two moles of hydrogen for each mole of lactic acid (Heiman et al., 2005):

\section{$\mathrm{CH}_{3} \mathrm{CHOHCOOH} \rightarrow \mathrm{CH}_{3} \mathrm{COCOOH}+\mathrm{H}_{2} \rightarrow \mathrm{CH}_{3} \mathrm{COOH}+\mathrm{H}_{2}$ Lactic acid Pyruvic acid Acetic acid}

The fermentation of lactic acid and its degradation products also generates bicarbonate ions $\left(\mathrm{HCO}_{3}{ }^{-}\right)$and hydrogen ions (Heiman et al., 2005; McCarty et al., 2007; Lee and Batchelor, 2004; Borum, 2002), thus increasing the alkalinity consumption of the dechlorination process.

Oxygen Release Compound (ORC) is a patented mixture of magnesium peroxide $\left(\mathrm{MgO}_{2}, 25-35 \% \mathrm{w} / \mathrm{w}\right)$, magnesium oxide ( $\mathrm{MgO}$, main component) and bi-acid potassium phosphate $\left(\mathrm{KH}_{2} \mathrm{PO}_{4}, 3 \% \mathrm{w} / \mathrm{w}\right)$. ORC appears as a fine $(\mathrm{d}<10 \mu \mathrm{m})$ white dust and may be employed by direct injection of a $40-60 \% \mathrm{w} / \mathrm{w}$ slurry or in a permeable reactive barrier. ORC degradative action is based on the controlled oxygen release, in a quantity equal to $10 \% \mathrm{w} / \mathrm{w}$ of the reagent, by magnesium peroxide at a contact with water and on the consequent support of biological oxidation of VOCs, with a significant increase of akalinity:

$\mathrm{MgO}_{2}+\mathrm{H}_{2} \mathrm{O} \rightarrow \frac{1}{2} \mathrm{O}_{2}+\mathrm{Mg}(\mathrm{OH})_{2}$

$\mathrm{MgO}+\mathrm{H}_{2} \mathrm{O} \rightarrow \mathrm{Mg}(\mathrm{OH})_{2}$

VOCs $+\mathrm{O}_{2} \rightarrow$ alcohols, organic acids $\rightarrow \mathrm{CO}_{2}+\mathrm{H}_{2} \mathrm{O}$

\section{MATERIALS AND METHODS}

All batch tests were carried out in $20 \mathrm{~mL}$ glass vials: an artificial aqueous phase, prepared by adding proper volumes of $1000 \mathrm{mg} \mathrm{L}^{-1}$ of nitrate, sulfate and chloride reference solutions (Fluka) to obtain the composition schematized in Table 1, was introduced in each vial, in presence of $1 \mathrm{~g}$ of not polluted soil and then the vial was sealed without any headspace by means of aluminum crimp caps and silicone/PTFE septa.

The soil, coming from a site in which a natural attenuation process concerning the considered pollutants occurs, has the aim to introduce degrading microorganisms in the system. Soil composition was investigated Table 2 by means of reference methods (Sparks, 1996). The sealed vials were then polluted by means of $20-60 \mu \mathrm{L}$ of VOCs standard solution injected through the septa and the batch tests were carried out by continuous turnover at $10 \mathrm{rpm}$,

Table 1: Physic-chemical characteristics of the artificial aqueous phase

$\mathrm{pH}=6.39, \mathrm{ORP}=450 \mathrm{mV}$

$\mathrm{Cl}^{-}=115 \mathrm{mg} \mathrm{L}^{-1}, \mathrm{SO}_{4}=5 \mathrm{mg} \mathrm{L}^{-1}, \mathrm{NO}_{3}{ }^{-}=1 \mathrm{mg} \mathrm{L}^{-1}$

Benzene $=1000 \mu \mathrm{g} \mathrm{L}^{-1}$, toluene $=2500 \mu \mathrm{g} \mathrm{L}^{-1}$,

Other VOCs $=500 \mu \mathrm{g} \mathrm{L}^{-1}$

Table 2: Physic-chemical characteristics of the soil

\begin{tabular}{|c|c|}
\hline Origin & $\begin{array}{l}\text { Depth } 21-24 \mathrm{~m} \text {, groundwater level } \\
: 13 \mathrm{~m} \text {, confined aquifer }\end{array}$ \\
\hline $\begin{array}{l}\text { Type } \\
\text { particle-size distribution } \\
\text { chemical properties }\end{array}$ & $\begin{array}{l}\text { clay with gypsum and carbonates } \\
\mathrm{d}<2 \mu \mathrm{m}: 57 \%, \mathrm{~d}<10 \mu \mathrm{m}: 80 \% \\
\text { pH } 7.50, \mathrm{CaCO}_{3} 7.9 \%, \mathrm{Organi}^{-} \text {Carbon } \\
0.38 \%, \mathrm{NO}_{3}^{-} 108.4 \mathrm{mg} \mathrm{kg}^{-1}, \mathrm{Cl}^{-} 41.2 \\
\mathrm{mg} \mathrm{kg}^{-1}, \mathrm{SO}_{4}{ }^{=} 11310 \mathrm{mg} \mathrm{kg}^{-1}\end{array}$ \\
\hline Metal contents (\%) & $\begin{array}{l}\mathrm{Na} 0.04, \mathrm{~K} 1.35, \mathrm{Ca} 15.09, \mathrm{Mg} 1.91 \\
\mathrm{Fe} 2.76, \mathrm{Al} 4.50, \mathrm{Ba} 0.02\end{array}$ \\
\hline Metal contents $(\mathrm{mg} / \mathrm{kg})$ & $\begin{array}{l}\text { Mn 590, Zn 116, Cd 5, Cr 107, Cu 34, Ni } \\
107, \mathrm{~Pb} 43, \text { Co } 14, \mathrm{Ti} 670, \mathrm{As} 36, \mathrm{Sb} 13\end{array}$ \\
\hline
\end{tabular}


with a total extent of 35-40 days and sampling intervals equal to 7 days, at which the aqueous phase underwent the determination of Oxidation Reduction Potential (ORP), $\mathrm{pH}$ and residual VOCs concentrations.

Three replicates for each sampling interval were analyzed and reference samples (blanks) were also considered to evaluate the eventual VOCs losses through the septa. Before the tests the release of COVs from the soil, keeping a solid/liquid ratio equal to 1:20 and considering a contact time equal to $48 \mathrm{~h}$, was verified to be absent: The detected organic compounds (long chain alcohols, aldheydes, kethones) were all related to the soil organic matter. The following VOCs reference solutions were employed in batch tests: Volatile Organic Compounds mix 2, $2000 \mu \mathrm{g} \mathrm{L} \mathrm{L}^{-1}$, Supelco (containing 13 compounds in methanol: Benzene, toluene, ethylbenzene, p-xylene, styrene, bromo benzene, 1, 3, 5trimetylbenzene, 1, 2, 4-trimetylbenzene.

P-isopropiltoluene, n-butilbenzene, 1, 2, 4trichlorobenzene, naftalene and 1, 2, 3-trichlorobenzene) was employed in the first and second tests, while 502/524.2. Volatile Organic Calibration Mix, $200 \mu \mathrm{g} \mathrm{mL}^{-1}$, Supelco (containing 60 compounds in methanol, among which the following 11 pollutants were considered: vinyl chloride, 1,1-dichloroethylene, 1,1-dichloroethane, 2,2dichloropropane, cis 1,2-dichloroethylene, benzene, trichloroethylene, toluene, etylbenzene, p-xylene, 1,4dichlorobenzene) was employed in the other tests. The VOCs contents were decided considering a polluted site in northern Italy, the same which gave origin to the soil employed in batch tests.

HRC was added in the first and second tests in a concentration equal to $3.3 \mathrm{~g} \mathrm{~L}^{-1}$ (Zanetti and Fiore, 2007) and ORC was added in the third test as a $10 \%$ $\mathrm{w} / \mathrm{w}$ suspension in water, corresponding to a theoretical oxygen release equal to 1000 times the stoichiometric amount necessary for the degradation of the pollutants contained in each vial.

At the beginning of the tests and for each sampling interval the aqueous phase was analyzed: chloride, nitrate and sulfate contents were detected by means of reference methods (Clesceri et al., 1998) and a UVVisible Unicam Elios $\alpha$ spectrophotometer; $\mathrm{pH}$ was measured employing an Orion 420 potentiometer and a glass $\mathrm{Ag} / \mathrm{AgCl}$ electrode; ORP was measured using a Orion SA520 potentiometer; VOCs contents were gathered by means of EPA8260B method EPA, through an Agilent GC/MS 7890-5975 equipped with a Gerstel CIS4 cryogenic injector and a HP5-MS column, employing static headspace injection.

The soil was sieved at $2 \mathrm{~mm}$ before the tests, to eliminate the coarse fraction. Total Organic Carbon content was detected by means of a Fisons TCM 480 carbon analyzer and metal contents were analyzed through acid digestion with hydrochloric and nitric acids in a Milestone 1200 Mega microwave oven and a Perkin-Elmer Optima 2000 ICP-OES spectrometer.

\section{RESULTS}

Table 2 contains the results of the characterization of the soil employed in the batch tests. The $\mathrm{pH}$ and ORP trends obtained from the batch tests are shown in Fig. 2.

The results of the performed batch tests are schematized in Fig 3, where all data series are compared and in Fig 4, where the degradation efficiency of HRC towards some CAHs is shown.

The experimental data gathered from the batch tests underwent a regression considering two different mathematical equations (a zero order model and a first order model) and the correspondent kinetic constant and half-life values were calculated Table 3 and 4. A zero order model represents a kinetic that is independent from the concentration of the pollutant removed from the aqueous phase, while a first order model is connected to a kinetic that is directly linked to the pollutant's concentration, implying a significant influence of the nature of the pollutant itself and therefore the existence of specific interactions between the substrate and the target compound.
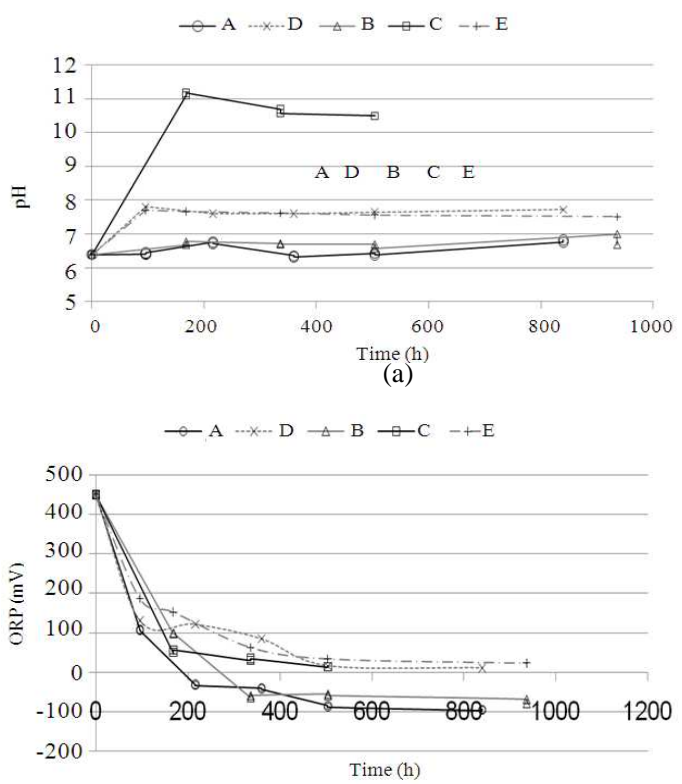

(b)

Fig. 2: $\mathrm{pH}$ and Oxidation-Reduction Potential (ORP) trends obtained from batch tests Data series: A. soil, HRC and BTEX + benzenic VOCs; B. soil, HRC and BTEX + CAHs; C. soil, ORC and BTEX + CAHs; D. soil and BTEX + benzenic VOCs; E. soil and BTEX + CAHs 
Am. J. Environ. Sci., 7 (5): 468-476, 2011

Table 3: Results of batch tests concerning BTEX: kinetic constant $(\mathrm{k})$ and half-life ( $\left.\mathrm{t}_{2}\right)$ values calculated applying zero order and first order kinetic models

\begin{tabular}{|c|c|c|c|c|c|c|}
\hline & \multicolumn{3}{|c|}{ Zero order model } & \multicolumn{3}{|c|}{ First order model } \\
\hline & $\mathrm{R}^{2}$ & $\mathrm{k}\left(\mathrm{h}^{-1}\right)$ & $t_{1 / 2}(h)$ & $\mathrm{R}^{2}$ & $\mathrm{k}\left(\mathrm{h}^{-1}\right)$ & $t_{1 / 2}(h)$ \\
\hline \multicolumn{7}{|c|}{ Benzene } \\
\hline A & 0.4645 & -0.4820 & 1037 & 0.5880 & -0.0008 & 866 \\
\hline B & 0.7191 & -0.4960 & 1008 & 0.8648 & -0.0009 & 770 \\
\hline $\mathrm{C}$ & 0.8774 & -1.5030 & 333 & 0.9816 & -0.0030 & 231 \\
\hline $\mathrm{E}$ & 0.8090 & -0.2573 & 1943 & 0.8436 & -0.0003 & 2310 \\
\hline \multicolumn{7}{|c|}{ Toluene } \\
\hline A & 0.3713 & -1.2290 & 1017 & 0.5432 & -0.0010 & 693 \\
\hline B & 0.4398 & -1.0372 & 1205 & 0.5615 & -0.0008 & 866 \\
\hline $\mathrm{C}$ & 0.6347 & -3.4497 & 362 & 0.7849 & -0.0030 & 231 \\
\hline $\mathrm{E}$ & 0.9493 & -1.6439 & 760 & 0.9695 & -0.0010 & 693 \\
\hline \multicolumn{7}{|c|}{ Ethylbenzene } \\
\hline A & 0.8078 & -0.3906 & 640 & 0.8352 & -0.0015 & 462 \\
\hline B & 0.9740 & -0.3845 & 650 & 0.9727 & -0.0013 & 533 \\
\hline $\mathrm{C}$ & 0.9791 & -0.7744 & 323 & 0.8563 & -0.0030 & 231 \\
\hline $\mathrm{E}$ & 0.9640 & -0.414 & 604 & 0.9570 & -0.0010 & 693 \\
\hline \multicolumn{7}{|c|}{ Xylene } \\
\hline A & 0.8348 & -0.4411 & 567 & 0.8329 & -0.0015 & 462 \\
\hline B & 0.9460 & -0.3023 & 827 & 0.9076 & -0.0010 & 693 \\
\hline $\mathrm{C}$ & 0.9886 & -0.7062 & 354 & 0.9480 & -0.0023 & 301 \\
\hline E & 0.9931 & -0.2642 & 946 & 0.9762 & -0.0007 & 990 \\
\hline
\end{tabular}

Table 4: Results of batch tests concerning some CAHs: kinetic constant $(\mathrm{k})$ and half-life $\left(\mathrm{t}_{\mathrm{t}}\right)$ ) values calculated applying zero order and first order kinetic models

\begin{tabular}{|c|c|c|c|c|c|c|}
\hline & \multicolumn{3}{|c|}{ Zero order model } & \multicolumn{3}{|c|}{ First order model } \\
\hline & $\mathrm{R}^{2}$ & $\mathrm{k}\left(\mathrm{h}^{-1}\right)$ & $t_{1 / 2}(h)$ & $\mathrm{R}^{2}$ & $\mathrm{k}\left(\mathrm{h}^{-1}\right)$ & $\mathrm{t}_{1 / 2}(\mathrm{~h})$ \\
\hline \multicolumn{7}{|l|}{ VC } \\
\hline B & 0.7148 & -0.2048 & 205 & 0.7176 & -0.0005 & 1386 \\
\hline $\mathrm{C}$ & 0.8233 & -0.2100 & 210 & 0.7963 & -0.0005 & 1386 \\
\hline E & 0.8319 & -0.1355 & 136 & 0.8296 & -0.0003 & 2310 \\
\hline \multicolumn{7}{|l|}{ 1,1-DCA } \\
\hline C & 0.6960 & -0.1499 & 150 & 0.6794 & -0.0003 & 2310 \\
\hline E & 0.9312 & -0.1021 & 102 & 0.9448 & -0.0002 & 3466 \\
\hline \multicolumn{7}{|l|}{ 1,1-DCE } \\
\hline $\begin{array}{l}\text { B } \\
\text { C }\end{array}$ & 0.9146 & -0.3751 & 375 & 0.9454 & -0.0013 & 533 \\
\hline $\begin{array}{l}\mathrm{C} \\
\mathrm{E}\end{array}$ & 0.9400 & -0.1600 & 160 & 0.9335 & -0.0003 & 2310 \\
\hline 1,2-DCE & 0.8726 & -0.0864 & 86 & 0.8801 & -0.0002 & 3466 \\
\hline B & 0.7381 & -0.3443 & 344 & 0.7959 & -0.0002 & 3466 \\
\hline $\mathrm{C}$ & 0.9629 & -0.1129 & 113 & 0.9589 & -0.0002 & 3466 \\
\hline E & 0.9515 & -0.1096 & 110 & 0.9478 & -0.0002 & 3466 \\
\hline \multicolumn{7}{|l|}{ TCE } \\
\hline $\mathrm{C}$ & 0.3245 & $\begin{array}{l}-0.40758 \\
-0.0758\end{array}$ & 76 & 0.3151 & $\begin{array}{l}-0.0017 \\
-0.0002\end{array}$ & $\begin{array}{r}400 \\
3466\end{array}$ \\
\hline E & 0.5317 & -0.0299 & 30 & 0.5363 & -0.00006 & 11552 \\
\hline \multicolumn{7}{|l|}{ dcP } \\
\hline B & 0.9835 & -0.3890 & 389 & 0.9910 & -0.0015 & 462 \\
\hline C & 0.8222 & -0.0898 & 90 & 0.8277 & -0.0002 & 3466 \\
\hline E & 0.8856 & -0.0439 & 44 & 0.8905 & -0.00009 & 7702 \\
\hline B & 0.9134 & -0.3988 & 399 & 0.9573 & -0.0014 & 495 \\
\hline $\mathrm{C}$ & 0.8948 & -0.1407 & 141 & 0.8810 & -0.0003 & 2310 \\
\hline E & 0.9615 & -0.0699 & 70 & 0.9573 & -0.0001 & 6931 \\
\hline
\end{tabular}

Data series: A. soil, HRC and BTEX + benzenic VOCs; B. soil, HRC and BTEX + CAHs; C. soil, ORC and BTEX + CAHs; E. soil and BTEX + CAHs. VOCs: VC: vinyl chloride; 1, 1-DCE: 1,1-dichloroethylene; 1,1-DCA: dichloroethane; 1,2-DCE: cis 1,2-dichloroethylene; TCE:

trichloroethylene; dcB: 1,4-dichlorobenzene; dcP: 2,2-dichloropropane

The purpose of the operation was to evaluate which kinetic model may reliably simulate the processes observed during the batch tests and to compare HRC and ORC performances, bearing in mind the net contribute of the soil to the removal of the pollutants from the aqueous phase. The data schematized in Table 3 concern the removal of BTEX by HRC, in absence/presence of CAHs and by ORC. The data reported in Table 4 are referred to the pollutants that were appreciably removed from the aqueous phase during the tests. 
Am. J. Environ. Sci., 7 (5): 468-476, 2011

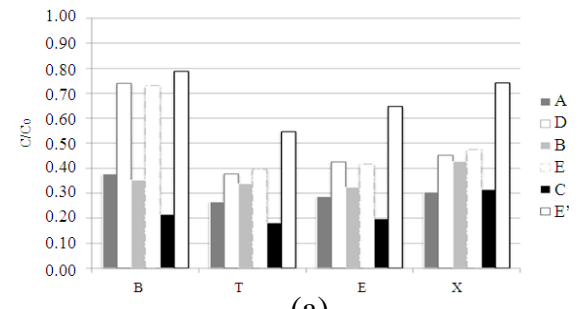

(a)

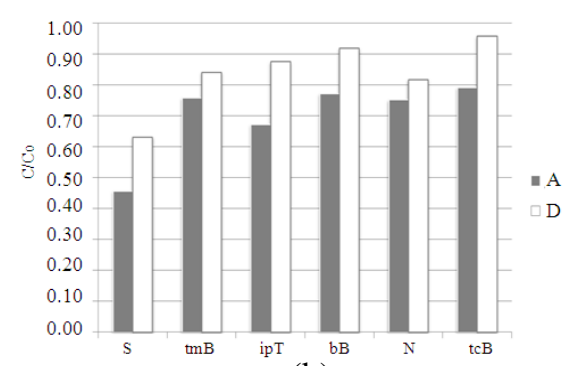

(b)

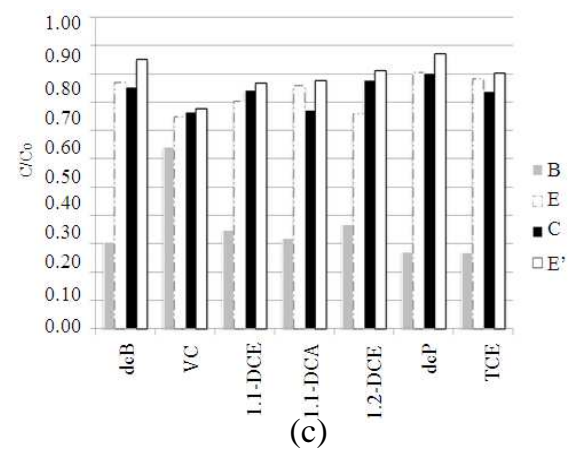

Fig. 3:Degradative efficiency of HRC and ORC evaluated from the results of batch tests Fig. 3 Legend Data series: A. soil, HRC and BTEX + benzenic VOCs (after 35 d); B. soil, HRC and BTEX + CAHs (after 39 d); C. soil, ORC and BTEX + CAHs (after 21 d); D. soil and BTEX + benzenic VOCs (after 35 d); E. soil and BTEX + CAHs (after 39 d); E'. soil and BTEX + CAHs (after 21 d) VOCs: B: benzene; T: toluene; E: ethylbenzene; $X$ : xylene; $S$ : styrene; tmB: trimethylbenzene; ipP: isopropyltoluene; bB: butylbenzene; $\mathrm{N}$ : naphthalene; tcB: 1,2,3trichlorobenzene; dcB: 1,4-dichlorobenzene; VC: vinyl chloride; 1,1-DCE: 1,1-dichloroethylene; 1,1-DCA: dichloroethane; 1,2-DCE: cis 1,2dichloroethylene; dcP: 2,2-dichloropropane; TCE: trichloroethylene

\section{DISCUSSION}

The soil exhibits a good buffering attitude in Fig $2 \mathrm{~A}$, because of the relevant content of carbonates Table 2. The ORP decreasing noticeable in Fig. $2 \mathrm{~B}$ may be linked to the metal oxides, particularly iron and manganese Table 2, able to take part in oxidationreduction reactions that involve the dissolved compounds in contact with soil particles surface (McBride, 1994). In all cases, both in presence and in absence of the reagents HRC and ORC, no relevant differences were observed in $\mathrm{pH}$ and ORP trends gathered from the two different pollutants groups.

HRC reagent's acidity opposes the alkalinity of the aqueous phase due to the soil Fig. 2A, while the molecular hydrogen released by lactic acid fermentation leads to moderately reducing conditions Fig. 2B. ORC reagent pushed the aqueous phase to $\mathrm{pH}$ values above 10 Fig. 2A; otherwise ORP trends resulted quite similar to the ones registered in absence of ORC Fig. 2B. ORC samples were not analyzed for periods above 21 days, because after this period the aqueous phase was not anymore in the vials, leaving behind just calcium hydroxide and the soil skeleton. The aqueous phase vanishing is a gradual event involving the batch tests employing ORC reagent, that is a very strong oxidant, able to react with water and the soil, leaving behind just its skeleton. Small gas bubbles were observed in the 20 $\mathrm{mL}$ vials about 24 hours after the beginning of the tests, then after 21 days a head-space equal to about $10 \mathrm{~mL}$ was detected, finally the aqueous phase completely disappeared after 28 days.

The degradative efficiency of HRC and ORC, expressed as the ratio between the final and initial concentration values of the pollutants gathered from the batch tests, are presented in Fig 2. BTEX were present in both groups of pollutants considered in the tests.

The comparison between $\mathrm{A}$ and $\mathrm{D}$ series and between $\mathrm{B}$ and $\mathrm{E}$ series underlines the net contribute of $\mathrm{HRC}$ in removing the pollutants from the aqueous phase: considering BTEX Fig 3A the only relevant contribute concerns benzene in both pollutants groups. The fact that benzene removal is higher in presence of CAHs and that an opposite trend is observed for toluene, ethylbenzene and xylene, suggests that benzene may take part in co-metabolic degradation processes involving CAHs, while toluene, ethylbenzene and xylene, probably compete with CAHs for HRC.

The ORC net degradation efficiency may be appreciated taking into account $\mathrm{C}$ and $\mathrm{E}$ ' data series (no relevant differences were detected for CAHs) and HRC and $\mathrm{ORC}$ reagents may be compared considering $\mathrm{B}$ and $\mathrm{C}$ data series Fig. 3C: The HRC's higher efficiency towards CAHs is clearly marked.

The data reported in Fig. 3C and the experimental data obtained from the batch tests involving HRC and CAHs (B data series), shown in Fig. 4, allow some comments about CAHs degradation: the results of the batch tests, considering the much higher degradative efficiency of HRC compared to ORC, give evidence of the reductive dechlorination. 
Am. J. Environ. Sci., 7 (5): 468-476, 2011

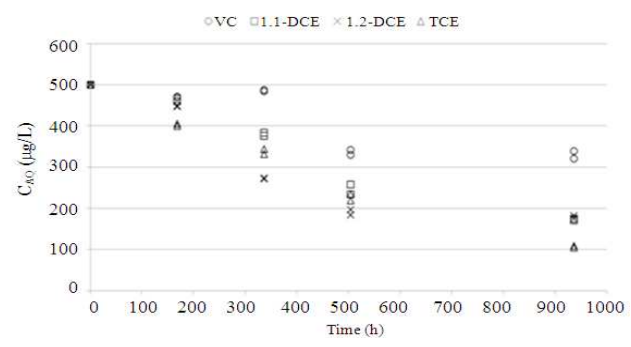

Fig. 4: Results of the batch tests: Degradative efficiency of HRC about some CAHs

The final concentrations of the CAHs and the higher C/Co values observed for 1, 2-DCE, 1,1-DCE, 1,1DCA and VC confirm both the dechlorination mechanism and the slower degradation of lesser chlorinated CAHs.

In view of BTEX Fig. 3A, ORC reagent exhibits a higher degradative efficiency if compared to HRC (see $\mathrm{B}$ and $\mathrm{C}$ data series), as expected that mono-aromatic pollutants are more sensitive to oxidation processes than to reduction ones (Farhadian et al., 2008). Considering the blanks Fig. 3A, D and E data, respectively referred to 35 and 39 days periods, are analogous, while E' data is referred to a 21 days period to make possible the comparison with $\mathrm{C}$ data.

As written before, the comparison of $\mathrm{A}$ and $\mathrm{B}$ data series allowed the evaluation of HRC degradative efficiency about BTEX in absence/presence of CAHs, for which BTEX may act as co-metabolic substrates. Otherwise the estimate of $\mathrm{B}$ and $\mathrm{C}$ data series allow the comparison of $\mathrm{HRC}$ and ORC reagents' efficiency and $\mathrm{E}$ data series outline the soil net contribute in removing the pollutants from the aqueous phase.

Taking into account BTEX Table 3, the only relevant differences between $\mathrm{A}$ and $\mathrm{B}$ data concern Benzene: $B$ data show higher $R^{2}$ values, particularly considering a first order kinetic model. $\mathrm{B}$ and $\mathrm{C}$ series experimental data concerning ethylene and xylene are equally reliably simulated by both kinetic models. Experimental data referred to toluene are the only ones for which just $\mathrm{E}$ series are consistently simulated by the considered kinetic models. In general, benzene and toluene experimental data show higher $\mathrm{R}^{2}$ values in the application of a first order model, while ethylene and xylene experimental data exhibit higher $\mathrm{R}^{2}$ values in the employment of a zero order model.

Considering CAHs Table 4, VC shows low $\mathrm{R}^{2}$ values for $\mathrm{B}$ data series, probably because of the dechlorination mechanism of high substituted compounds that enhances $\mathrm{VC}$ concentration with a kinetic higher than the one regarding $\mathrm{VC}$ dechlorination. $\mathrm{C}$ and $\mathrm{E}$ data series about VC show higher $\mathrm{R}^{2}$ values, particularly applying a zero order kinetic model. $\mathrm{C}$ and $\mathrm{E}$ data series about the other CAHs considered in Table 4 exhibit higher $\mathrm{R}^{2}$ values for a zero order model, while $\mathrm{B}$ data series show higher $\mathrm{R}^{2}$ values for a first order model. $E$ data series display equivalent $R^{2}$ values for both the applied kinetic models.

\section{CONCLUSION}

This study has the aim of a preliminary study to evaluate the potential of two innovative reagents, one able to release oxygen (ORC) and another able to release hydrogen (HRC), in the degradation of some VOCs. On the grounds of the obtained results the following issues may be underlined:

- Oxygen Release Compound (ORC) exhibits a good degradation efficiency towards BTEX and a scarce degradation efficiency about Chlorinated Aliphatic compounds (CAHs)

- Hydrogen Release Compound (HRC) demonstrated a good degradation efficiency about Chlorinated Aliphatic compounds (CAHs) and benzene

- Taking into account the experimental results concerning HRC in absence of CAHs (A data series), both zero order and first order models were not a reliable simulation about benzene and toluene, though considering ethylene and xylene the two models revealed equivalent trustworthiness

- Experimental results concerning HRC in presence of CAHs (B data series) were reliably approximated by a first order kinetic model taking into account all the studied pollutants, with the only exception of xylene, for which a zero order kinetic model was more consistent and toluene, for which no reliable approximation of experimental results was found

- Experimental results referred to ORC (C data series) are consistent both with a zero order kinetic model and with a first order kinetic model about CAHs, ethylene and xylene and with a first order kinetic model about benzene and toluene

\section{ACKNOWLEDGMENT}

The experimental data presented in this study were obtained in the Environmental Chemistry Laboratory at DITAG, Politecnico di Torino, Italy and all the analytical procedures were performed by the authors. The authors declare that no competing financial interests exist about the topic presented in this study. 
Am. J. Environ. Sci., 7 (5): 468-476, 2011

\section{REFERENCES}

Adamson, D.T., D.Y. Lyon and J.B. Hughes, 2004. Flux and product distribution during biological treatment of tetrachloroethene dense non-aqueousphase liquid. Environ. Sci. Technol., 38: 20212028. PMID: 15112802

Amos, B.K., J.A. Christ, L.M. Abriola, K.D. Pennell and F.E. Loffler, 2007. Experimental evaluation and mathematical modeling of microbially enhanced tetrachloroethene (PCE) Dissolution. Environ. Sci. Technol., 41: 963-970. DOI: 10.1021/es061438n.

Clesceri, L.S., A.D. Eaton and A.E. Greenberg, 1998. Standard Methods for the Examination of Water and Wastewater. 20th Ed., American Public Health Association, Washington, pp: 1220.

Aulenta, F., A. Bianchi, M. Majone, M.P. Papini and M. Potalivo et al., 2005. Assessment of natural or enhanced in situ bioremediation at a chlorinated solvent-contaminated aquifer in Italy: A microcosm study. Environ. Int., 31: 185-190. DOI: 10.1016/j.envint.2004.09.014

Aulenta, F., M. Majone and V. Tandoi, 2006. Enhanced anaerobic bioremediation of chlorinated solvents: Environmental factors influencing microbial activity and their relevance under field conditions. J. Chem. Technol. Biotechnol, 81: 1463-1474. DOI: $10.1002 /$ jctb. 1567

Aulenta, F., A. Pera, S. Rossetti, M.P. Papini and M. Majone, 2007. Relevance of side reactions in anaerobic reductive dechlorination microcosms amended with different electron donors. Water Res. 41: 27-38. DOI: 10.1016/j.watres.2006.09.019

Azizian, M.F., I.P.G. Marshall, S. Behrens, A.M. Spormann and L. Semprini, 2010. Comparison of lactate, formate and propionate as hydrogen donors for the reductive dehalogenation of trichloroethene in a continuous-flow column. J. Contam. Hydrol., 113: 77-92. DOI: 10.1016/j.jconhyd.2010.02.004

Borum, E., 2002. Bioremediation of chlorinated solvents in fractured bedrock: Characterization and case studies, US Environmental Protection Agency.

Christ, J.A., C.A. Ramsburg, L.M. Abriola, K.D. Pennell and F.E. Loffler, 2005. Coupling aggressive mass removal with microbial reductive dechlorination for remediation of dnapl source zones: a review and assessment. Environ. Health Perspect., 113: 465-477. PMID: 15811838

Cope, N. and J.B. Hughes, 2001. BiologicallyEnhanced removal of PCE from NAPL source zones. Environ. Sci. Technol., 35: 2014-2021. DOI: $10.1021 / \mathrm{es} 0017357$
EPA, 2009. Hydrogen Release Compund (HRC®) Barrier Application at the North of Basin F Site, Rocky Mountain Arsenal: Innovative Technology Evaluation Report. United State Enviornment Protection Agency.

Farhadian, M., C. Vachelard, D. Duchez and C. Larroche, 2008. In situ bioremediation of monoaromatic pollutants in groundwater: A review, Bioresour. Technol. 99: 5296-5308. DOI: 10.1016/j.biortech.2007.10.025

Ferguson, J.F. and J.M.H. Pietari, 2000. Anaerobic transformations and bioremediation of chlorinated solvents. Environ. Poll., 107: 209-215. DOI: 10.1016/S0269-7491(99)00139-6

Frascari, D., D. Pinelli, M. Nocentini, A. Zannoni and S. Fedi et al., 2006. Long-term aerobic cometabolism of a chlorinated solvent mixture by vinyl chloride-, methane- and propane-utilizing biomasses. J. Hazard. Mater., 138: 29-39. $\quad$ DOI: 10.1016/j.jhazmat.2006.05.009

Frascari, D., A. Zannoni, D. Pinelli and M. Nocentini, 2007. Chloroform aerobic cometabolism by butane-utilizing bacteria in bioaugmented and nonbioaugmented soil/groundwater microcosms. Proc. Biochem., $\quad 42$ : 1218-1228. DOI: 10.1016/j.procbio.2007.05.018

Grunanger, P. and P.V. Finzi, 1997. Chimica Organica. 6th Edn., CEA, Milano, ISBN: 8840808922, pp: 1376.

Heimann, A.C., A.K. Friis and R. Jakobsen, 2005. Effects of sulfate on anaerobic chloroethene degradation by an enriched culture under transient and steady-state hydrogen supply. Water Res. 39: 3579-3586. DOI: 10.1016/j.watres.2005.06.029

Lee, W. and B. Batchelor, 2004. Abiotic reductive dechlorination of chlorinated ethylenes by ironbearing phyllosilicates. Chemosphere 56 : 9991009. DOI: $10.1016 /$ j.chemosphere.2004.05.015

McBride, M.B., 1994. Environmental Chemistry of Soils. 1st Edn., Oxford University Press, New York, ISBN: 0195070119, pp: 406.

McCarty, P.L., 1993. In situ bioremediation of chlorinated solvents. Curr. Op. Biotechnol. 4: 323330. DOI: 10.1016/0958-1669(93)90103-4

McCarty, P.L., M.Y. Chu and P.K. Kitanidis, 2007. Electron donor and $\mathrm{pH}$ relationships for biologically enhanced dissolution of chlorinated solvent DNAPL in groundwater. Eur. J. Soil Biol. 43: 276-282. DOI: 10.1016/j.ejsobi.2007.03.004

Middeldorp, P.J.M., M.L.G.C. Luijten, B.A.V.D. Pas, M.H.A.V. Eekert and S.W.M. Kengen et al., 1999. Anaerobic microbial reductive dehalogenation of chlorinated ethenes. Bioremediat. J., 3: 151-169. DOI: $\quad 10.1080 / 10889869991219280$ 
Robinson, C., D.A. Barry, P.L. McCarty, J.I. Gerhard and I. Kouznetsova, 2009. pH control for enhanced reductive bioremediation of chlorinated solvent source zones. Sci. Total Environ. 407: 4560-4573. DOI: 10.1016/j.scitotenv.2009.03.029

Silva, M.L.D., R.C. Daprato, D.E. Gomez, J.B. Hughes and C.H. Ward et al., 2006. Comparison of bioaugmentation and biostimulation for the enhancement of dense nonaqueous phase liquid source zone bioremediation. Water Environ. Res., 78: 2456-2465. PMID: 17243245

Sparks, D.L., 1996. Methods of Soil Analysis. Part 3, Chemical Methods. 1st Edn., Soil Science Society of America, Madison, ISBN-10: 0891188258, pp: 1390.
Yang, Y. and P.L. McCarty, 2002. Comparison between donor substrates for biologically enhanced tetrachloroethene DNAPL dissolution. Environ. Sci. Technol., 36: 3400-3404. DOI: 10.1021/es011408e

Yang, Y.Y. and P.L. McCarty, 1998. Competition for hydrogen within a chlorinated solvent dehalogenating anaerobic mixed culture. Environ. Sci. Technol., 32: 3591-3597. DOI: 10.1021/es980363n

Zanetti, M.C. and S. Fiore, 2007. Lab tests for the remediation of a PCE polluted site by means of HRC Reactant. Am. J. Environ. Sci., 3: 151-157. DOI: 10.3844/ajessp.2007.151.157 